\title{
Molecular imaging of fibrin in a breast cancer xenograft mouse model
}

\author{
Ritika Uppal ${ }^{1,2}$, Zdravka Medarova $^{1}$, Christian T. Farrar ${ }^{1}$, Guangping Dai ${ }^{1}$, Anna Moore ${ }^{1}$, \\ and Peter Caravan ${ }^{1}$ \\ ${ }^{1}$ Athinoula A. Martinos Center for Biomedical Imaging, Department of Radiology, Massachusetts \\ General Hospital and Harvard Medical School, Charlestown, MA 02129
}

\section{Abstract}

Rationale and objectives-Fibrin deposition has been indicated within the stroma of a majority of solid tumors. Here we assess the feasibility of using the established fibrin-specific probe EP-2104R for the noninvasive imaging of fibrin in the context of breast cancer.

Methods-EP-2104R, untargeted Gd-DTPA and a newly synthesized non-fibrin binding control linear peptide (CLP) were compared using steady-state and dynamic contrast enhanced MR imaging in a breast cancer xenograft mouse model at 9.4T.

Results-EP-2104R transiently enhanced both the tumor core and periphery, but only the enhancement in the tumor periphery persisted even 90 min after EP-2104R administration. However, untargeted Gd-DTPA and CLP are not retained in the tumor periphery. The half-life of EP-2104R in the tumor periphery $(103 \pm 18 \mathrm{~min})$ is significantly longer $(\mathrm{p}<0.05)$ than either GdDTPA (29.6 $\pm 2.4 \mathrm{~min})$ or CLP $(42.4 \pm 1.5 \mathrm{~min})$, but the rate of clearance is similar for all the three probes from the tumor core. The presence of high concentrations of fibrin in the tumor periphery was corroborated using immuno-histochemistry with a fibrin-specific antibody.

Conclusions-The persistent enhancement observed in the tumor periphery with EP-2104R is likely a result of its fibrin-specific binding rather than its size and demonstrates the feasibility of EP-2104R for molecular imaging of fibrin in tumor stroma.

\section{Keywords}

fibrin; tumor stroma; EP-2104R; gadolinium; breast cancer

\section{Introduction}

\begin{abstract}
In 1865, Trosseau observed that thromboembolic complications were a common occurrence in cancer patients and suggested that there must be a correlation between the hemostatic system and cancer biology.1 Since then, numerous studies have proved a wound-like process to be an essential requirement for tumorigenesis. ${ }^{2,3}$ Several clinical studies have shown that the expression of procoagulants and fibrinolytic factors by tumor cells and/or stromal cells correlates with advanced disease and poor outcome for cancer. ${ }^{4}$ The deposition of fibrin by various cancer forms, especially solid tumors is well established. ${ }^{5-9}$ The existence of a heterogenous pattern of fibrin/fibrinogen deposition within the stroma of various tumor types is supported by techniques such as immunofluorescence and immunoelectron microscopy and points to a potential role for fibrin in tumor stroma
\end{abstract}

\footnotetext{
${ }^{2}$ Current address: GE Healthcare, Medical Diagnostics, Bangalore, India

The other authors have no disclosures.
} 
formation and in localizing fibrin and fibrinogen to the tumor-host cell interface. ${ }^{10-12}$ Moreover, the fibrin matrix is known to promote migration of cells such as transformed cells, macrophages and fibroblasts. In particular, deposition of fibrin along with other adhesive glycoproteins into the extracellular matrix supports binding to growth factors and promotes the cellular response to adhesion, proliferation, migration during angiogenesis and tumor cell growth. ${ }^{7,10,13,14}$ Furthermore, a correlation exists between the plasma fibrinogen levels and tumor size, depth of tumor invasion and metastasis. ${ }^{15,16}$ It is also known that fibrin is involved in protecting tumor cells from the circulating natural killer cells of the immune system. ${ }^{17}$

Thus fibrin may represent a useful biomarker for solid tumors and metastases in general, and tumor fibrin concentration may correlate with tumor aggressiveness. Molecular imaging of fibrin offers the potential to identify and characterize tumors. There have been several MR probes reported that target fibrin, ${ }^{18-26}$ the fibrin-fibronectin complex, ${ }^{27-30}$ as well as other coagulation components. ${ }^{31}$ For imaging fibrin in the tumor interstitium, ideally the probe should be small enough to rapidly extravasate and bind to fibrin in the matrix, and small peptide-based probes meet this criterion.

Here we investigate whether the fibrin-specific probe EP-2104R can specifically identify fibrin in a breast cancer xenograft mouse model. EP-2104R comprises a cyclic peptide for fibrin binding conjugated to four GdDOTA moieties for MR signal enhancement. ${ }^{22}$ EP-2104R was shown to be efficacious in identifying thrombi in the heart chambers, arteries or veins in animal models and in clinical trials. ${ }^{32-43}$ More recently EP-2104R was investigated in a rat glioma model. ${ }^{44}$ Here, we compare the tumor enhancing characteristics of EP-2104R to those of untargeted controls. Part of this work was communicated previously. ${ }^{45}$

\section{Materials and Methods}

All experiments were performed in accordance with the NIH Guide for the Care and Use of Laboratory Animals and were approved by the institution's animal care and use committee. The human adenocarcinoma BT-20 cell line was provided by ATCC (Manassas, VA). The cell line was maintained in Eagle's Minimum Essential Medium (ATCC, Manassas, VA), supplemented with fetal bovine serum to a final concentration of $10 \%$, as suggested by the supplier of the cell line. Cells of the same passage number were used for all experiments. Tumors were grown in the right flank of 5-6 week old nude mice by subcutaneous injection of $3 \times 10^{6}$ BT-20 cells in $50 \mu \mathrm{L}$ of HBSS. The tumor size was monitored and animals were used for imaging when the tumor size was $3-5 \mathrm{~mm}$ in diameter. This time period was typically two weeks.

EP-2104R was a gift from Epix Pharmaceuticals (Lexington, MA, USA). Gadopentetate dimeglumine (Gd-DTPA) was purchased from Bayer Healthcare, (Montville, NJ, USA). GdDTPA was used as a control since untargeted extracellular agents like Gd-DTPA are routinely used clinically and pre-clinically to characterize tumors based on permeability changes. EP-2104R contains a cyclic (disulfide linkage) peptide. As a further control, we prepared a control linear peptide (CLP) version of EP-2104R that has the same molecular size and relaxation properties, but does not bind to fibrin. Cyclic EP-2104R was reduced and the free cysteines were alkylated with iodoacetic acid using a literature procedure. ${ }^{46}$ EP-2104R (20.4 mg, $4.4 \mu \mathrm{mol})$ was dissolved in $2 \mathrm{~mL}$ of reduction buffer $(0.2 \mathrm{M}$ Tris, 2.5 $\mathrm{mM}$ EDTA and $6 \mathrm{M}$ guanidine-HCl, $\mathrm{pH} \mathrm{8.5)} \mathrm{and} 10.2 \mathrm{mg}$ of dithiothreitol (DTT, $66 \mu \mathrm{mol}$, 15 eq) was added to the solution while nitrogen was passed over the solution. The solution was incubated at $37{ }^{\circ} \mathrm{C}$ for $3 \mathrm{~h}$ followed by cooling on ice. Iodoacetic acid (35 mg) was dissolved in $0.35 \mathrm{~mL}$ alkylation buffer (1.5 M Tris, $2.5 \mathrm{mM}$ EDTA and 6M guanidine- $\mathrm{HCl}$, 
$\mathrm{pH} 8.5)$ to prepare a $0.54 \mathrm{M}$ solution. An aliquot of the iodoacetic solution $(0.134 \mathrm{~mL}, 72$ $\mu \mathrm{mol}, 16.3 \mathrm{eq}$ ) was added to the cold peptide solution and allowed to sit for 20 min under a stream of nitrogen. Mercaptoethanol $(0.12 \mathrm{~mL})$ was then added and the mixture was allowed to sit for an additional $10 \mathrm{~min}$. The $\mathrm{pH}$ was decreased to 2 with $\mathrm{HCl}$ and then the reaction mixture was applied to a $\mathrm{C} 18$ sep-pak column and the peptide was eluted from the column using $20 \% \mathrm{MeCN} / 80 \% \mathrm{H}_{2} \mathrm{O}$. The fractions collected were analyzed on a $\mathrm{C} 4$ reverse phase column on an analytical HPLC with UV and MS detection and the pure fractions were combined. The experimental yield was $16.8 \mathrm{mg}(82 \%)$. HPLC analysis showed purity >98\%; electrospray MS (+ mode): $m / z$ expected $\left[\mathrm{C}_{158} \mathrm{H}_{224} \mathrm{ClGd}_{4} \mathrm{~N}_{34} \mathrm{O}_{60} \mathrm{~S}_{2}+2 \mathrm{H}\right]^{2+} 2144.08$, found 2144.0.

Fibrin binding of EP-2104R and CLP were assessed in a plate-based fibrin binding assay described previously. ${ }^{22,47}$ Briefly, fibrinogen solutions $(2.5 \mathrm{mg} / \mathrm{mL})$ were aliquoted into 96 well plates, thrombin and calcium chloride were added to induce clotting to fibrin, and the resultant gels were dried at $37{ }^{\circ} \mathrm{C}$ overnight. These fibrin gels were rehydrated with increasing concentrations of probe and incubated for $2 \mathrm{~h}$ at $37{ }^{\circ} \mathrm{C}$ in microtiter wells containing $7 \mu \mathrm{M}$ fibrin (based on fibrinogen monomer). Then the concentration of probe in the supernatant (unbound probe) was assayed using ICP-MS to quantify gadolinium. The concentration of bound probe was calculated as [bound] $=$ [total] - [unbound] .

MR imaging was performed at 9.4T using a small animal scanner (Biospec, Bruker Biospin, Billerica, MA) using a homemade $30 \times 19 \mathrm{~mm}$ oval surface coil. Mice were placed prone in a cradle with the head secured in a stereotaxic frame. Anesthesia was maintained with isoflurane $\left(1-1.5 \%\right.$ in $\left.100 \% \mathrm{O}_{2}\right)$ and the mouse was kept warm by blowing warm air on the animal. The tail vein was cannulated for intravenous delivery of the contrast agent while the animal was positioned in the scanner.

The imaging paradigm involved baseline $\mathrm{T} 2$ weighted imaging to localize the tumor, followed by dynamic T1-weighted imaging prior to and immediately following probe administration. The T1-weighted imaging was repeated out to 90 minutes post injection to track washout. The FOV for all the MRI sequences was $3.2 \times 3.2 \mathrm{~cm}$. For dynamic imaging we used multislice FLASH (TR/TE/flip angle $=100 \mathrm{~ms} / 1.92 \mathrm{~ms} / 60^{\circ}$ ). For the initial bolus, matrix was $128 \times 128$ with 1 average giving a temporal resolution of $13 \mathrm{~s}$ and this was repeated for $13 \mathrm{~min}$. At later time points we imaged at higher resolution with more averages (TR/TE/flip=100/2.3/60, matrix $256 \times 256,16$ averages, acquisition time $=7 \mathrm{~min})$.

Mice were imaged when the tumor had grown to a diameter of 3-5 mm. We imaged 12 mice in total. In 10 of these mice, after the baseline scans were completed, $50 \mu \mathrm{L}$ of control probe (Gd-DTPA, $200 \mu \mathrm{mol} / \mathrm{kg}$ or CLP, $20 \mu \mathrm{mol} / \mathrm{kg}$ ) was injected as a bolus via the tail vein and the dynamic contrast enhancement was observed using the low resolution multi-slice FLASH images. This was followed by a series of high-resolution multi-slice FLASH collected for $90 \mathrm{~min}$. Subsequently, EP-2104R $(20 \mu \mathrm{mol} / \mathrm{kg})$ or CLP $(20 \mu \mathrm{mol} / \mathrm{kg})$ was injected as a bolus via the tail vein and the same imaging sequence was repeated for $90 \mathrm{~min}$ post injection. The relaxivities of Gd-DTPA and EP-2104R at $9.4 \mathrm{~T}$ are $3.7 \mathrm{mM}^{-1} \mathrm{~s}^{-1}$ and 25.6 (6.4 per $\mathrm{Gd}) \mathrm{mM}^{-1} \mathrm{~s}^{-1}$, respectively, ${ }^{48,49}$ based on its similar size we expect a similar relaxivity to EP-2104R for CLP. Six mice received Gd-DTPA followed by EP-2104R, three mice received Gd-DTPA followed by CLP, and one mouse received CLP followed by EP-2104R. An additional two mice received only EP-2104R and were scanned for 120 minutes post injection.

Images were analyzed using ImageJ by drawing ROIs and measuring signal intensity (SI) in the tumor core, tumor periphery and adjacent muscle in the same slice. Tumor periphery here is defined as the regions on the border of the tumor that showed different signal 
washout kinetics compared to the central tumor core. These were regions that showed persistent enhancement post EP-2104R injection. For each animal, the same ROIs were used for both imaging probes. Noise was quantified as the standard deviation (SD) of the signal measured in the air adjacent to the animal. Contrast to noise ratios (CNR) were calculated for the difference between tumor (core or periphery) and muscle using equation 1.

$$
\begin{gathered}
\mathrm{CNR}(\text { tumor } / \text { muscle })=[\mathrm{SI}(\text { tumor core or periphery }) \mathrm{SI}(\text { muscle })] / \mathrm{SD}(\text { air }) \\
\qquad \begin{array}{c}
\mathrm{CNR}(\mathrm{t})=\mathrm{CNR}(0) * \exp (\mathrm{k} * \mathrm{t}) \\
\mathrm{t}_{1 / 2}=\ln (2) / \mathrm{k}
\end{array}
\end{gathered}
$$

Contrast agent washout of the tumor core and tumor periphery was estimated from a monoexponential fit of the CNR vs time curves, equation 2, where $\mathrm{CNR}(\mathrm{t})$ is the $\mathrm{CNR}$ at time $t, \operatorname{CNR}(0)$ is the peak $\mathrm{CNR}$, and $k$ is the rate constant for washout. $\mathrm{CNR}(0)$ and $k$ were iteratively varied to produce the best fit to the data. The rate constants were used to generate half-lives $\left(\mathrm{t}_{1 / 2}\right)$ for contrast agent washout as given by equation 3 .

Data are expressed as \pm SEM and were compared using a either or paired t-test or a twosided t-test with Bonferroni correction for multiple comparisons as appropriate, with $\mathrm{p}<0.05$ considered significant.

After the animals were sacrificed, the tumors were excised and frozen. $5 \mu \mathrm{m}$-thick cryosections were mounted on slides and immunohistochemistry was performed using monoclonal fibrin antibody (1:25 dilution; product \# NYBT2G1, Accurate Chemical \& Scientific Corp., Westbury, NY) using the manufacturer's recommended protocol: $1 \mathrm{~h}$ at room temperature with the horse radish peroxidase conjugated antibody, followed by diaminobenzidine (DAB) for 5 min. Finally, sections were counterstained with hematoxylin.

\section{Results}

The linear peptide control (CLP) was readily prepared by reduction of the disulfide bond in EP-2104R followed by alkylation of the cysteine thiols with iodoacetic acid, Figure 1 . The binding of CLP to fibrin was assessed in a direct binding assay. No binding to fibrin could be observed under the conditions of the study ( $7 \mu \mathrm{M}$ fibrin, $5-50 \mu \mathrm{M}$ CLP) indicating the cyclic structure of the peptide is essential for fibrin binding. EP-2104R was used as a positive control in this assay and we obtained a $\mathrm{Kd}$ of $1.8 \mu \mathrm{M}$ for EP-2104R in excellent agreement with a previous reported study $(\mathrm{Kd}=1.7 \mu \mathrm{M})$.

In all 12 animals, the tumors grew $3-5 \mathrm{~mm}$ in diameter after 2 weeks. We initially imaged two mice prior to, and immediately following EP-2104R i.v. injection. As expected, administration of EP-2104R resulted in positive enhancement of the tumors, but we also observed differences in signal washout between the tumor core and periphery. To address potential complications due to tumor heterogeneity and other sources of inter-animal variability, we decided to perform intra-animal comparisons among Gd-DTPA, EP-2104R, and CLP, by using sequential administration of two probes. Representative MR images showing enhancement and washout of probe for injection of Gd-DTPA and subsequent injection of EP-2104R are shown in Figure 2 and images of a mouse that received Gd-DTPA followed by CLP are shown in Figure 3. We noted that there was a difference in the kinetics of contrast enhancement between the circular tumor core and regions on the border of the tumor. This spatial difference in contrast enhancement between core and periphery was 
observed for all three probes. We defined regions of interest for the tumor core (ROI that covered the entire circular cross section), tumor periphery (typically two triangle shaped ROI on each side of the tumor), and adjacent muscle, as identified in Figures 2 and 3. For each animal, the same ROIs were used for characterizing enhancement with both injected probes. For all three probes, the tumor core was initially strongly enhanced followed by a rapid washout period (Figure $4 \mathrm{a}$ and $4 \mathrm{c}$ ). The kinetics of contrast washout from the tumor core was similar for all three probes. However, the kinetics of contrast washout from the tumor periphery was different among all three probes. From Figure $4 \mathrm{~b}$ and $4 \mathrm{~d}$ it is apparent that EP-2104R stays in the tumor periphery much longer than Gd-DTPA or CLP.

In 6 mice we injected Gd-DTPA and imaged until this compound had cleared and signal had approached baseline levels (90 minutes) and then injected EP-2104R and imaged for another 90 minutes. We compared washout from the tumor core and tumor periphery using the same ROI for both probes in a pair-wise fashion. The washout of EP-2104R was 20\% slower than Gd-DTPA from the tumor core but this small difference in rates was not significant $\left(\mathrm{t}_{1 / 2}=\right.$ $38 \pm 9$ for Gd-DTPA vs $45 \pm 9$ min for EP-2104R, $p=0.17$ ). In these animals, washout of EP-2104R was 190 $\pm 75 \%$ slower than Gd-DTPA from the tumor periphery $(\mathrm{p}<0.05)$.

In the next 3 mice we compared the washout of the linear peptide CLP to Gd-DTPA from the tumor periphery. In these 3 animals, washout of CLP was $77 \pm 18 \%$ slower than GdDTPA from the tumor periphery $(\mathrm{p}<0.05)$. This result suggested that part of the retention of EP-2104R in the tumor periphery was due to its larger size. The washout of CLP was slower than Gd-DTPA from the tumor core but this difference in rates was not significant $\left(\mathrm{t}_{1 / 2}=\right.$ $28 \pm 5$ for Gd-DTPA vs $46 \pm 21$ min for CLP, $p=0.35$ ). To confirm that the retention of EP-2104R in the tumor periphery has a specific component, we directly compared EP-2104R and CLP in one mouse. In that animal the half-life in the tumor periphery was $38.7 \mathrm{~min}$ for CLP but $201 \mathrm{~min}$ for EP-2104R.

The half-life measurements for all animals are shown in Table 1. Comparison of the halflives of Gd-DTPA or CLP versus EP-2104R in the tumor periphery indicates that both GdDTPA and CLP have significantly shorter than the half-lives of EP-2104R ( $<<0.05$ for both). Additionally, the relative standard deviation (RSD) for Gd-DTPA and CLP half-life measurements was low, while the RSD for EP-2014R was 52\%. Greater heterogeneity in the EP-2104R half-lives might be expected since the fibrin content is likely to vary among animals.

We also compared the increase in CNR at 85 minutes post injection ( $\triangle \mathrm{CNR}$ ) relative to the baseline CNR $\left(\mathrm{CNR}_{\text {pre }}\right)$. At this timepoint the blood concentration is expected to be close to zero. For EP-2104R, $\Delta \mathrm{CNR} / \mathrm{CNR}_{\mathrm{pre}}=7.2 \pm 2.9$ for the tumor periphery and this value was significantly higher than for Gd-DTPA $(2.6 \pm 0.8, \mathrm{p}<0.05)$. In the cohort where we compared Gd-DTPA to CLP, the increase in tumor:muscle CNR at 85 minutes was low and not significantly different $(\triangle \mathrm{CNR} / \mathrm{CNRpre}=1.0 \pm 0.8$ for Gd-DTPA and $0.6 \pm 1.8$ for CLP).

Immunohistochemistry of frozen tissue sections (Figure 5) derived from the tumor (left) or adjacent muscle (right) were visualized under light microscopy. Distinct fibrin-rich areas (arrow) could be identified at the periphery of the tumor, but much lower levels of fibrin were seen in the tumor core. By contrast, DAB enhancement in the muscle tissue was at background levels.

\section{Discussion}

This study demonstrates prolonged enhancement within the peripheral stroma of subcutaneous tumors in a breast cancer xenograft mouse model after the injection of EP-2104R. Signal washout from the tumor periphery was noticeably slower than washout 
from the tumor core. Immunohistochemical staining for fibrin indicated much denser staining for fibrin in the periphery of the tumor than within the tumor core.

A particular challenge in molecular imaging of tumors is in determining whether the probe shows a truly specific enhancement. The endothelial dysfunction associated with tumors results in increased permeability, and this is exploited daily in the clinic where non-specific extracellular contrast agents like Gd-DTPA are used to identify tumors. ${ }^{50}$ Tumor contrast enhancement has been reported with a range of non-specific probes ranging from small molecules like Gd-DTPA to medium sized dendrimer-based compounds to large nanoparticles. ${ }^{51,52}$ We anticipated that EP-2104R would also show general, non-specific enhancement of the tumor and this was indeed observed. In order to separate non-specific enhancement from enhancement due to fibrin binding, we made intra-animal comparisons of tumor enhancement using first Gd-DTPA and then EP-2104R. We chose the intra-animal paradigm to account for differences in tumor heterogeneity and cardiac output from mouse to mouse. Thus all the comparisons are matched because they are performed among the different regions within the same animal, and paired tests can be used for all comparisons. By reducing biologic variability in this way, statistical power is increased for a given sample size. Because Gd-DTPA clears rapidly, we could first image a mouse with Gd-DTPA and then inject either EP-2104R or CLP in the same single scanning session.

There was no significant difference in washout from the tumor core between Gd-DTPA and EP-2104R and this suggests that the concentration of fibrin in the core is below the limit of detection by EP-2104R at 9.4T. The similarity in washout rates may be expected since it was shown that EP-2104R like Gd-DTPA has only very low affinity for blood plasma proteins and EP-2104R undergoes exclusive renal excretion like Gd-DTPA. ${ }^{22,33}$ Thus clearance in the absence of fibrin binding is governed only by the tumor permeability and the glomerular filtration rate, which will be the same for an intra-animal comparison.

On the other hand we observed a large and significant difference in washout from the tumor periphery between Gd-DTPA and EP-2104R. This difference was attributed to fibrin binding in the periphery. This was qualitatively supported by immunohistochemical staining for fibrin, where fibrin levels were much higher in the periphery. We also noted that the washout of Gd-DTPA from the periphery was very similar among all the mice whereas there was more variability in the washout of EP-2104R from the tumor periphery. Within the tumor periphery there was heterogeneity in the rate of EP-2104R washout. We chose ROIs that covered the whole tumor periphery, but it was obvious in some mice that clearance of EP-2104R varied several fold across this ROI. This heterogeneity in washout for EP-2104R is also suggestive of fibrin binding, as one would expect differences in fibrin concentrations among the animals and within the tumor itself.

EP-2104R has a molecular weight of $4300 \mathrm{Da}$, which is an order of magnitude higher than Gd-DTPA. In order to address whether Gd-DTPA is an appropriate control, we synthesized a non-binding analog of EP-2104R. The cyclic peptide of EP-2104R is critical for fibrin binding. To develop a size matched, non-binding control (termed CLP) we reduced the disulfide bond of EP-2104R and capped the cysteine thiols with iodoacetic acid to prevent re-formation of the disulfide linkage. In an intra-animal comparison, the washout of CLP from tumor periphery was significantly slower than Gd-DTPA. However comparing CLP to EP-2104R across all animals, EP-2104R was still significantly longer retained in the tumor periphery. Head to head comparison of EP-2104R and CLP in the same animal showed similar washout of the tumor core but 5-fold slower washout of EP-2104R from the tumor periphery. Taken together, these results suggest a specific enhancement of fibrin within the tumor stroma. 
The extended enhancement observed in the tumor periphery after injection of EP-2104R may be a general steady-state marker for solid tumors, although this needs to be validated in additional models. Further work should address the utility of this probe in other cancer models. It would also be worthwhile to determine whether fibrin binding could increase specificity in distinguishing malignant versus benign lesions. For instance in the context of breast cancer it is not clear whether there is significant fibrin present in fibroadenoma, fibrotic cysts, or post-operative scar that could confound diagnosis. Quantitative analysis of fibrin in different lesion types is warranted. It would also be useful to determine whether molecular imaging of fibrin correlates with tumor aggressiveness. Although it was not addressed in this study, it is apparent that the non-specific dynamic enhancement of the tumor could be exploited to estimate $\mathrm{K}^{\text {trans }}$, the volume transfer coefficient that is often used to characterize tumor permeability. ${ }^{53}$ Steady state imaging of fibrin could be combined with dynamic estimates of permeability to better characterize the tumor microenvironment.

There are some limitations to this work. Our study was performed at 9.4T and the relaxivity of fibrin-bound EP-2104R is much lower at this field compared to clinical field strengths. ${ }^{22,48}$ The limit of detection of fibrin would be expected to be higher at $1.5 \mathrm{~T}$ or $3 \mathrm{~T}$ where the relaxivity of the probe is increased. A second limitation is that we performed only one intra-animal study with both CLP and EP-2104R. However on a group basis, we demonstrated that EP-2104R is retained significantly longer in the tumor periphery than either Gd-DTPA or CLP.

\section{Conclusion}

This work demonstrates the feasibility of EP-2104R for molecular imaging of fibrin in tumor stroma. As with any Gd-based probe, there is a dynamic non-specific enhancement of the tumor. However the kinetics of contrast enhancement are such that fibrin-based enhancement can be distinguished on delayed, steady state images after the non-specific component has washed out from the tumor.

\section{Acknowledgments}

This work was supported in part by award R01HL109448 from the National Heart, Lung, and Blood Institute, award R01EB009062 from the National Institute of Biomedical Imaging and Bioengineering, and by awards P41RR14075 and S10RR025563 from the National Center for Research Resources. P.C. has equity in Factor 1A, LLC, the company which holds the patent rights to EP-2104R.

\section{References}

1. Trosseau, A. Clinique medicale de l'Hotel-Dieu de Paris. Bailliere, JB., editor. Vol. 3. New Sydenham Society; London: 1865. p. 654-712.

2. Schuh AC, Keating SJ, Monteclaro FS, et al. Obligatory wounding requirement for tumorigenesis in v-jun transgenic mice. Nature. 1990; 346:756-760. [PubMed: 2167455]

3. Dolberg DS, Hollingsworth R, Hertle M, et al. Wounding and its role in RSV-mediated tumor formation. Science. 1985; 230:676-678. [PubMed: 2996144]

4. Palumbo JS, Degen JL. Mechanisms linking tumor cell-associated procoagulant function to tumor metastasis. Thromb Res. 2007; 120(Suppl 2):S22-28. [PubMed: 18023710]

5. Bardos H, Molnar P, Csecsei G, et al. Fibrin deposition in primary and metastatic human brain tumours. Blood Coagul Fibrinolysis. 1996; 7:536-548. [PubMed: 8874864]

6. Costantini V, Zacharski LR. Fibrin and cancer. Thromb Haemost. 1993; 69:406-414. [PubMed: 8322261]

7. Dvorak HF, Nagy JA, Berse B, et al. Vascular permeability factor, fibrin, and the pathogenesis of tumor stroma formation. Ann N Y Acad Sci. 1992; 667:101-111. [PubMed: 1309029] 
8. Cavanaugh PG, Sloane BF, Honn KV. Role of the coagulation system in tumor-cell-induced platelet aggregation and metastasis. Haemostasis. 1988; 18:37-46. [PubMed: 3047022]

9. Dvorak HF. Thrombosis and cancer. Hum Pathol. 1987; 18:275-284. [PubMed: 3546076]

10. Rickles FR, Patierno S, Fernandez PM. Tissue factor, thrombin, and cancer. Chest. 2003; 124:58S68S. [PubMed: 12970125]

11. Simpson-Haidaris PJ, Rybarczyk B. Tumors and fibrinogen. The role of fibrinogen as an extracellular matrix protein. Ann N Y Acad Sci. 2001; 936:406-425. [PubMed: 11460495]

12. Costantini V, Zacharski LR, Memoli VA, et al. Fibrinogen deposition without thrombin generation in primary human breast cancer tissue. Cancer Res. 1991; 51:349-353. [PubMed: 1670992]

13. Brown LF, Van de Water L, Harvey VS, et al. Fibrinogen influx and accumulation of cross-linked fibrin in healing wounds and in tumor stroma. Am J Pathol. 1988; 130:455-465. [PubMed: 3279791]

14. Dvorak HF, Harvey VS, Estrella P, et al. Fibrin containing gels induce angiogenesis. Implications for tumor stroma generation and wound healing. Lab Invest. 1987; 57:673-686. [PubMed: 2447383]

15. Lee JH, Ryu KW, Kim S, et al. Preoperative plasma fibrinogen levels in gastric cancer patients correlate with extent of tumor. Hepatogastroenterology. 2004; 51:1860-1863. [PubMed: 15532844]

16. Palumbo JS, Kombrinck KW, Drew AF, et al. Fibrinogen is an important determinant of the metastatic potential of circulating tumor cells. Blood. 2000; 96:3302-3309. [PubMed: 11071621]

17. Palumbo JS, Talmage KE, Massari JV, et al. Platelets and fibrin(ogen) increase metastatic potential by impeding natural killer cell-mediated elimination of tumor cells. Blood. 2005; 105:178-185. [PubMed: 15367435]

18. Flacke S, Fischer S, Scott MJ, et al. Novel MRI contrast agent for molecular imaging of fibrin implications for detecting vulnerable plaques. Circulation. 2001; 104:1280-1285. [PubMed: 11551880]

19. Winter PM, Caruthers SD, Yu X, et al. Improved molecular imaging contrast agent for detection of human thrombus. Magn Reson Med. 2003; 50:411-416. [PubMed: 12876719]

20. Winter PM, Cai K, Chen J, et al. Targeted PARACEST nanoparticle contrast agent for the detection of fibrin. Magn Reson Med. 2006; 56:1384-1388. [PubMed: 17089356]

21. Nair S, Kolodziej AF, Bhole G, et al. Monovalent and Bivalent Fibrin-specific MRI Contrast Agents for Detection of Thrombus. Angew Chem Int Ed Engl. 2008; 47:4918-4921. [PubMed: 18496805]

22. Overoye-Chan K, Koerner S, Looby RJ, et al. EP-2104R: A fibrin-specific gadolinium-based MRI contrast agent for detection of thrombus. Journal of the American Chemical Society. 2008; 130:6025-6039. [PubMed: 18393503]

23. Pan D, Senpan A, Caruthers SD, et al. Sensitive and efficient detection of thrombus with fibrinspecific manganese nanocolloids. Chem Commun (Camb). 2009:3234-3236. [PubMed: 19587924]

24. Zhang Z, Kolodziej AF, Qi J, et al. Effect of Peptide-Chelate Architecture on Metabolic Stability of Peptide-based MRI Contrast Agents. New J Chem. 2010; 2010:611-616.

25. Pan D, Caruthers SD, Senpan A, et al. Synthesis of NanoQ, a copper-based contrast agent for highresolution magnetic resonance imaging characterization of human thrombus. J Am Chem Soc. 2011; 133:9168-9171. [PubMed: 21599030]

26. Zhang Z, Kolodziej AF, Greenfield MT, et al. Heteroditopic binding of magnetic resonance contrast agents for increased relaxivity. Angew Chem Int Ed Engl. 2011; 50:2621-2624. [PubMed: 21370351]

27. Pilch J, Brown DM, Komatsu M, et al. Peptides selected for binding to clotted plasma accumulate in tumor stroma and wounds. Proc Natl Acad Sci U S A. 2006; 103:2800-2804. [PubMed: 16476999]

28. Ye F, Wu X, Jeong EK, et al. A peptide targeted contrast agent specific to fibrin-fibronectin complexes for cancer molecular imaging with MRI. Bioconjug Chem. 2008; 19:2300-2303. [PubMed: 19053180] 
29. Tan M, Wu X, Jeong EK, et al. Peptide-targeted Nanoglobular Gd-DOTA monoamide conjugates for magnetic resonance cancer molecular imaging. Biomacromolecules. 2010; 11:754-761. [PubMed: 20131758]

30. Tan M, Wu X, Jeong EK, et al. An effective targeted nanoglobular manganese(II) chelate conjugate for magnetic resonance molecular imaging of tumor extracellular matrix. Mol Pharm. 2010; 7:936-943. [PubMed: 20481565]

31. Duerschmied D, Meibetaner M, Peter K, et al. Molecular magnetic resonance imaging allows the detection of activated platelets in a new mouse model of coronary artery thrombosis. Invest Radiol. 2011; 46:618-623. [PubMed: 21577120]

32. Vymazal J, Spuentrup E, Cardenas-Molina G, et al. Thrombus imaging with fibrin-specific gadolinium-based MR contrast agent EP-2104R: results of a phase II clinical study of feasibility. Invest Radiol. 2009; 44:697-704. [PubMed: 19809344]

33. Uppal R, Catana C, Ay I, et al. Bimodal Thrombus Imaging: Simultaneous PET/MR Imaging with a Fibrin-targeted Dual PET/MR Probe--Feasibility Study in Rat Model. Radiology. 2011; 258:812-820. [PubMed: 21177389]

34. Uppal R, Ay I, Dai G, et al. Molecular MRI of intracranial thrombus in a rat ischemic stroke model. Stroke. 2010; 41:1271-1277. [PubMed: 20395615]

35. Stracke CP, Katoh M, Wiethoff AJ, et al. Molecular MRI of cerebral venous sinus thrombosis using a new fibrin-specific MR contrast agent. Stroke. 2007; 38:1476-1481. [PubMed: 17379818]

36. Spuentrup E, Katoh M, Wiethoff AJ, et al. Molecular magnetic resonance imaging of pulmonary emboli with a fibrin-specific contrast agent. Am J Resp Crit Care Med. 2005; 172:494-500. [PubMed: 15937292]

37. Spuentrup E, Katoh M, Buecker A, et al. Molecular MR imaging of human thrombi in a swine model of pulmonary embol! ism usin $\mathrm{g}$ a fibrin-specific contrast agent. Investigative Radiology. 2007; 42:586-595. [PubMed: 17620942]

38. Spuentrup E, Fausten B, Kinzel S, et al. Molecular magnetic resonance imaging of atrial clots in a swine model. Circulation. 2005; 112:396-399. [PubMed: 16009790]

39. Spuentrup E, Buecker A, Katoh M, et al. Molecular magnetic resonance imaging of coronary thrombosis and pulmonary emboli with a novel fibrin-targeted contrast agent. Circulation. 2005; 111:1377-1382. [PubMed: 15738354]

40. Spuentrup E, Botnar RM, Wiethoff AJ, et al. MR imaging of thrombi using EP-2104R, a fibrinspecific contrast agent: initial results in patients. European Radiology. 2008; 18:1995-2005. [PubMed: 18425519]

41. Botnar RM, Buecker A, Wiethoff AJ, et al. In vivo magnetic resonance imaging of coronary thrombosis using a fibrin-binding molecular magnetic resonance contrast agent. Circulation. 2004; 110:1463-1466. [PubMed: 15238457]

42. Sirol M, Fuster V, Badimon JJ, et al. Chronic thrombus detection with in vivo magnetic resonance imaging and a fibrin-targeted contrast agent. Circulation. 2005; 112:1594-1600. Epub 2005 Sep 1596. [PubMed: 16145001]

43. Katoh M, Haage P, Wiethoff AJ, et al. Molecular Magnetic Resonance Imaging of Deep Vein Thrombosis Using a Fibrin-Targeted Contrast Agent: A Feasibility Study. Invest Radiol. 2009; 44:146-150. [PubMed: 19151606]

44. Morelli JN, Runge VM, Williams JM, et al. Evaluation of a fibrin-binding gadolinium chelate peptide tetramer in a brain glioma model. Invest Radiol. 2011; 46:169-177. [PubMed: 21150792]

45. Uppal R, Medarova Z, Farrar C, et al. Intl Soc Magn Reson Med. 2009:1250.

46. Simpson, RJ. Proteins and Proteomics: A Laboratory Manual. Cold Spring Harbor Laboratory Press; Cold Spring Harbor, New York: 2003.

47. Kolodziej A, Nair SA, Graham PB, et al. Fibrin Specific Peptides Derived by Phage Display: Characterization of Peptides and Conjugates for Imaging. Bioconjug Chem. 2012

48. Caravan P, Farrar CT, Frullano L, et al. Influence of molecular parameters and increasing magnetic field strength on relaxivity of gadolinium- and manganese-based T1 contrast agents. Contrast Media Mol Imaging. 2009; 4:89-100. [PubMed: 19177472] 
49. Nieminen MT, Rieppo J, Silvennoinen J, et al. Spatial assessment of articular cartilage proteoglycans with Gd-DTPA-enhanced T1 imaging. Magn Reson Med. 2002; 48:640-648. [PubMed: 12353281]

50. Essig M, Weber MA, von Tengg-Kobligk H, et al. Contrast-enhanced magnetic resonance imaging of central nervous system tumors: agents, mechanisms, and applications. Top Magn Reson Imaging. 2006; 17:89-106. [PubMed: 17198225]

51. Turetschek K, Preda A, Novikov V, et al. Tumor microvascular changes in antiangiogenic treatment: assessment by magnetic resonance contrast media of different molecular weights. J Magn Reson Imaging. 2004; 20:138-144. [PubMed: 15221819]

52. Orth RC, Bankson J, Price R, et al. Comparison of single- and dual-tracer pharmacokinetic modeling of dynamic contrast-enhanced MRI data using low, medium, and high molecular weight contrast agents. Magn Reson Med. 2007; 58:705-716. [PubMed: 17899608]

53. Tofts PS, Brix G, Buckley DL, et al. Estimating kinetic parameters from dynamic contrastenhanced T(1)-weighted MRI of a diffusable tracer: standardized quantities and symbols. J Magn Reson Imaging. 1999; 10:223-232. [PubMed: 10508281] 

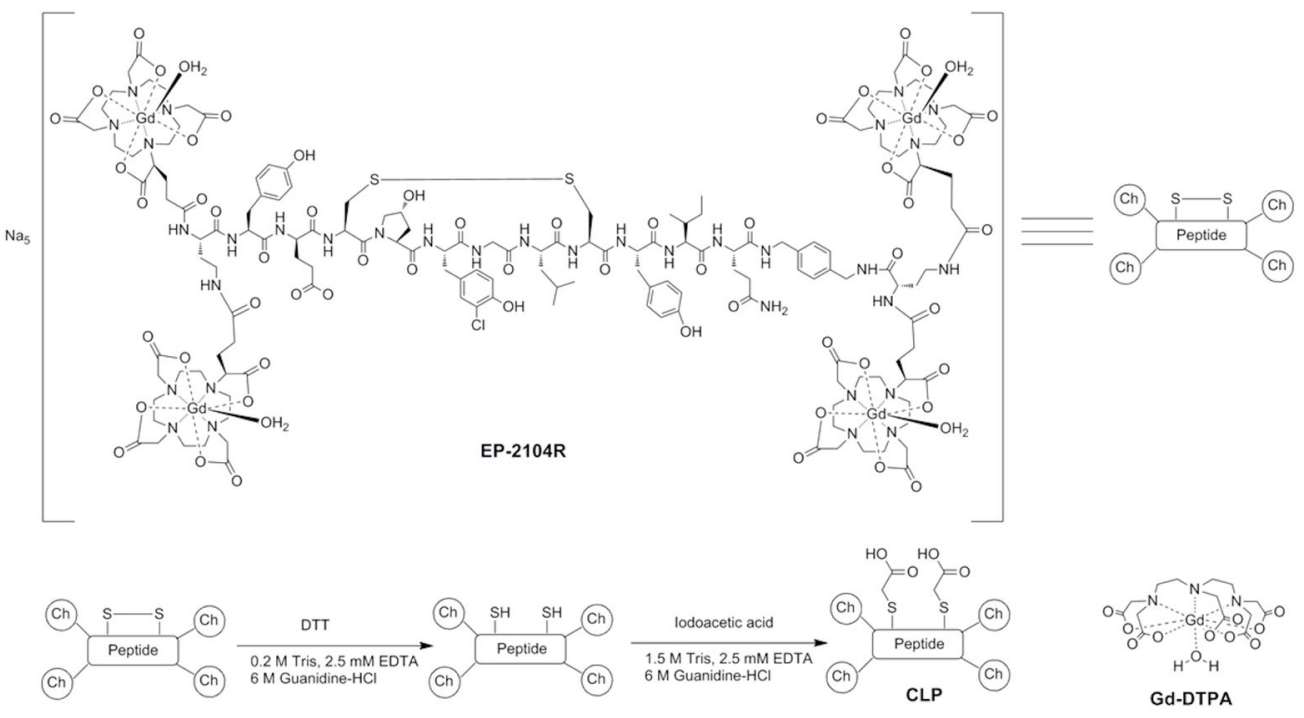

Figure 1.

Chemical structures of EP-2104R, Gd-DTPA and scheme for synthesis of the control linear peptide (CLP) from EP-2104R. 


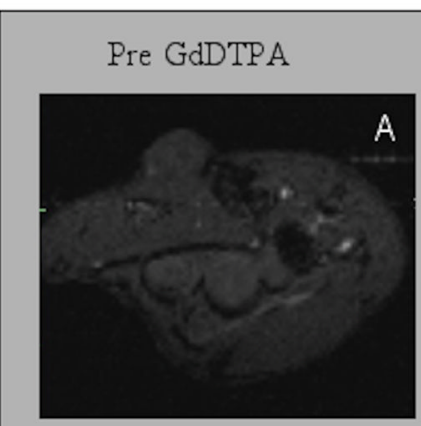

Pre EP-2104R

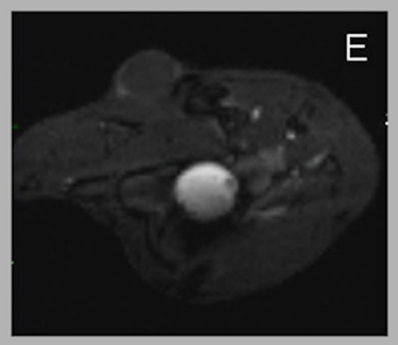

\section{$0.2^{\prime}$ post GdDTPA}

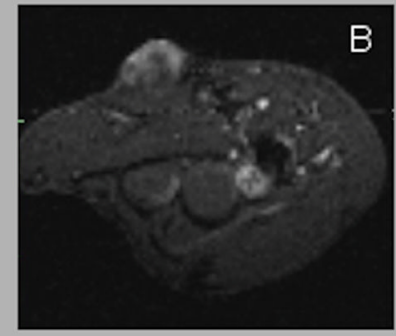

$0.2^{\prime}$ post EP-2104R

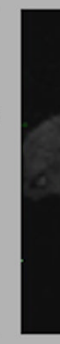

$15^{\prime}$ post GdDTPA

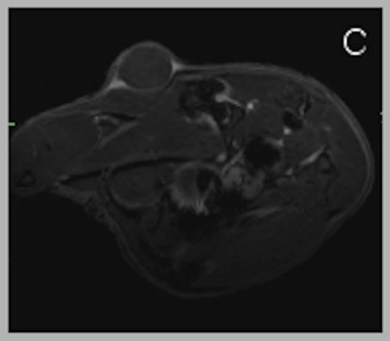

$15^{\prime}$ post EP-2104R

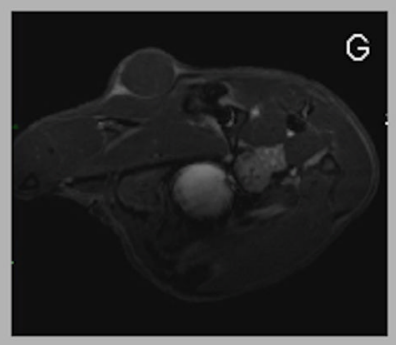

$99^{\prime}$ post GdDTPA

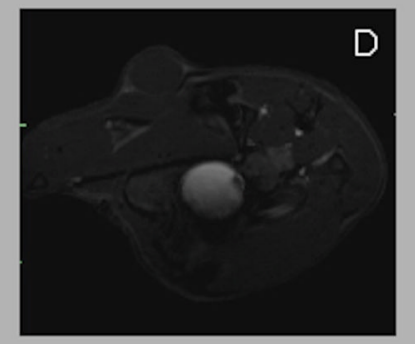

$92^{\prime}$ post EP-2104R

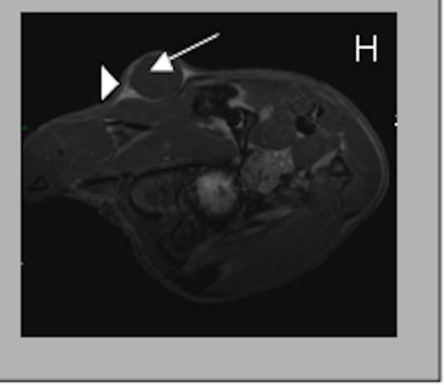

Figure 2.

Serial FLASH images pre- and post-injection of Gd-DTPA (top) and EP-2104R (bottom) show transient tumor core enhancement following contrast administration. The tumor periphery remains enhanced at least 90 min after EP-2104R injection. Low resolution images A, B, E and F. High resolution images C, D, G and H. Arrow in image H indicates the tumor core and arrowhead indicates triangular enhanced region in tumor periphery. 


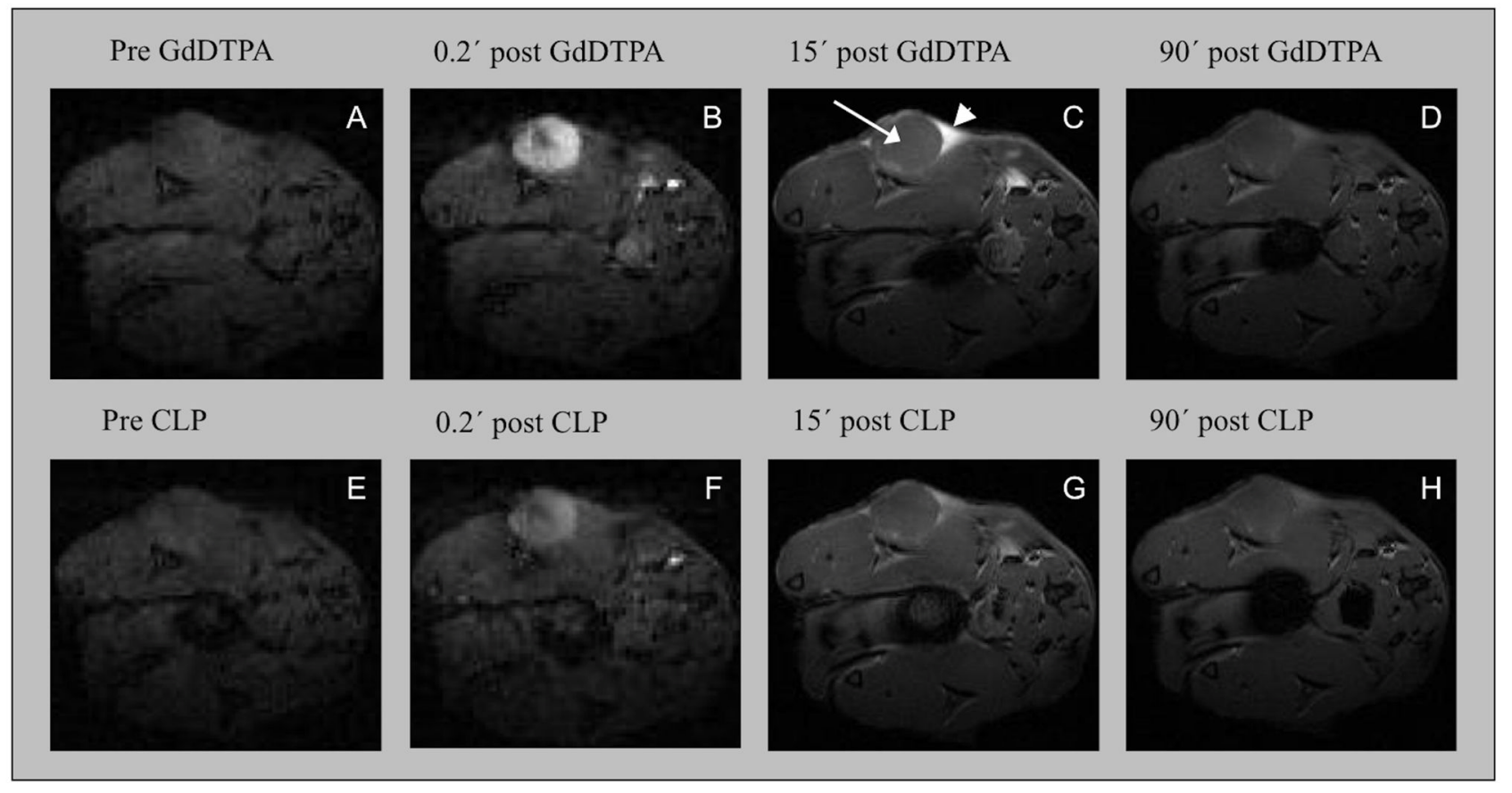

Figure 3.

Serial FLASH images pre- and post-injection of Gd-DTPA (top) and CLP (bottom) show transient tumor core enhancement following contrast administration, while regions of the tumor periphery show different enhancement kinetics. Arrow in image $\mathrm{C}$ indicates the tumor core and arrowhead indicates triangular enhanced region in tumor periphery. Washout of CLP from the periphery is 77\% slower than Gd-DTPA due to its larger size, but both compounds show significantly faster washout than EP-2104R. Low resolution images A, B, $\mathrm{E}$ and $\mathrm{F}$. High resolution images $\mathrm{C}, \mathrm{D}, \mathrm{G}$ and $\mathrm{H}$. 

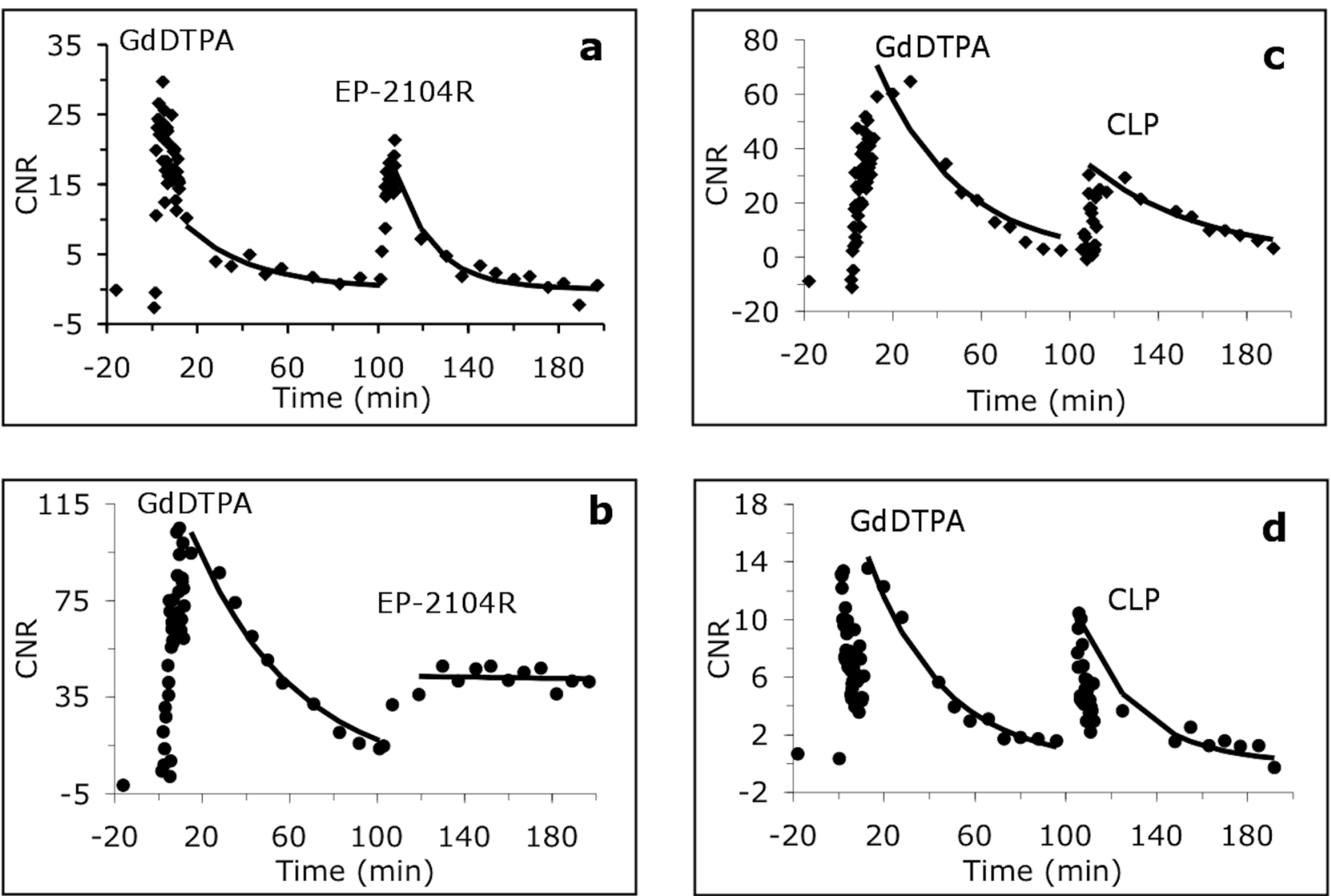

Figure 4.

CNR of the tumor core (top row, panels (a) and (c)) and of the tumor periphery (bottom row, panels (b) and (d)) relative to muscle. Panels (a) and (b) show data for injection of GdDTPA followed by EP-2104R in the same animal. Panels (c) and (d) show data for injection of GdDTPA followed by CLP in the same animal. 


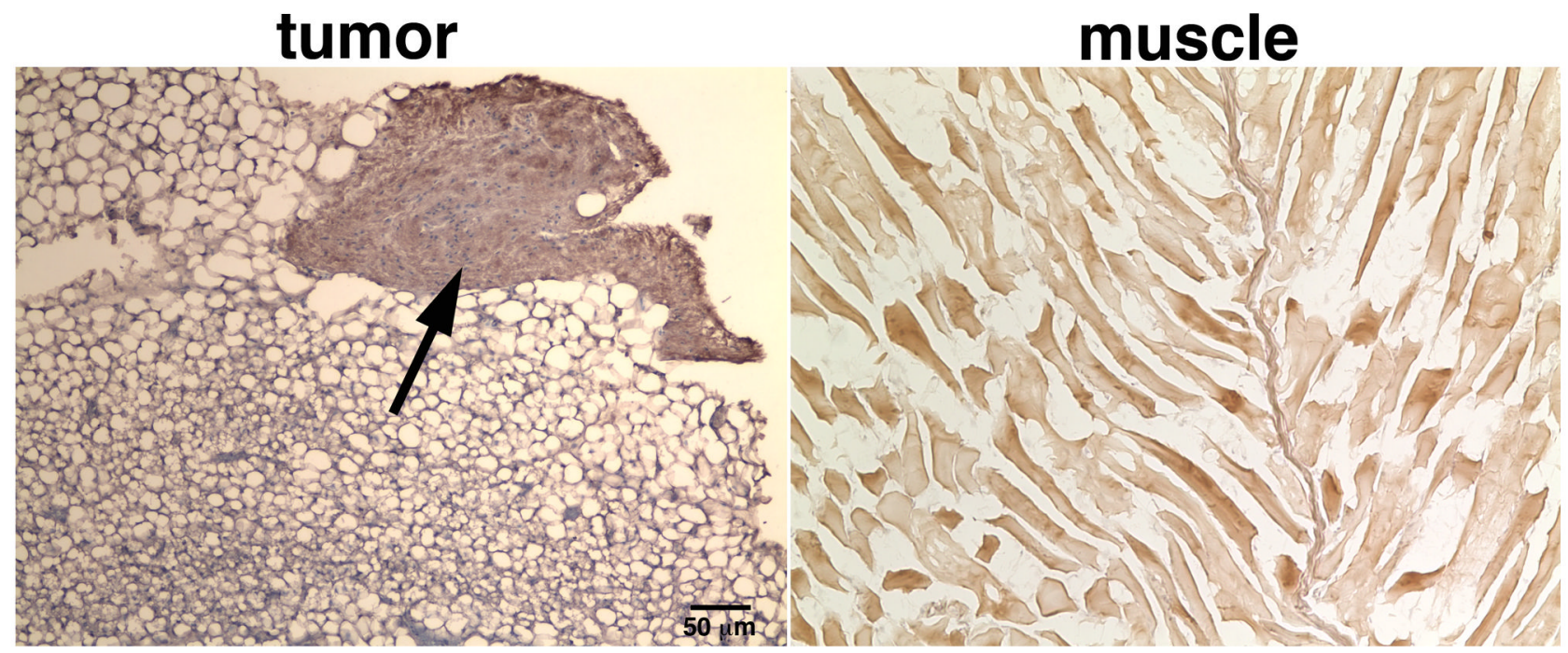

Figure 5.

Representative images of frozen tissue sections derived from the tumor (left) and adjacent muscle (right). The sections were stained with an HRP-conjugated fibrin-specific antibody, counterstained with DAB (brown) and visualized under light microscopy. Distinct fibrinrich areas (arrow) could be identified at the periphery of the tumor. By contrast, DAB enhancement in the muscle tissue was at background levels. 


\section{Table 1}

Half-life (min) of each imaging probe in the tumor periphery as estimated from CNR vs time curves for all the mice imaged in this study. The half-life of EP-2104R in the tumor periphery is significantly $(\mathrm{p}<0.05)$ longer than either Gd-DTPA or CLP.

\begin{tabular}{|r|r|r|r|}
\hline Animal & $\mathbf{t}_{\mathbf{1} / \mathbf{2}}$ GdDTPA & $\mathbf{t}_{\mathbf{1} / \mathbf{2}} \mathbf{E P}$-2104R & $\mathbf{t}_{\mathbf{1} / \mathbf{2}}$ CLP \\
\hline 1 & & 114 & \\
\hline 2 & & 94 & \\
\hline 3 & 27 & 160 & \\
\hline 4 & 38 & 121 & \\
\hline 5 & 26 & 94 & \\
\hline 6 & 23 & 32 & \\
\hline 7 & 38 & 60 & \\
\hline 8 & 41 & 53 & \\
\hline 9 & 29 & & 44 \\
\hline 10 & 20 & & 42 \\
\hline 11 & 27 & & 45 \\
\hline 12 & & 201 & 39 \\
\hline Mean (St Dev) & $30(7)$ & $103(53)$ & $42(3)$ \\
\hline & & & \\
\hline
\end{tabular}

